\title{
Codonolactone, a sesquiterpene lactone isolated from Chloranthus henryi Hemsl, inhibits breast cancer cell invasion, migration and metastasis by downregulating the transcriptional activity of Runx2
}

\author{
WEI WANG ${ }^{1 *}$, BIN CHEN $^{1 *}$, RUOLAN ZOU $^{2}$, XIUYING TU $^{1}$, SONGLIN TAN $^{1}$, \\ HONG LU ${ }^{3}$, ZHAOJIE LIU ${ }^{1}$ and JIANJIANG FU ${ }^{1}$

\begin{abstract}
${ }^{1}$ Department of Pharmacology, School of Pharmacy, Jiangxi University of Traditional Chinese Medicine, Nanchang 330004;
${ }^{2}$ Hospital of Nanchang University, Nanchang 330047; ${ }^{3}$ Network and Educational Technology Center, Jiangxi University of Traditional Chinese Medicine, Nanchang 330004, P.R. China
\end{abstract}

Received March 28, 2014; Accepted June 10, 2014

DOI: $10.3892 /$ ijo.2014.2643

\begin{abstract}
Metastasis is the most insidious aspect of breast cancer, but effective strategies to control this malignant process are still lacking. In previous studies, we screened over 200 extracts from plants of genus Chloranthaceae by bioactivity-guided fractionation, and found that Codonolactone (CLT) exhibited potential antimetastatic properties in breast cancer cells. This sesquiterpene lactone was isolated from Chloranthus henryi Hemsl, and is also found in other medical herbs, such as Codonopsis pilosula, Atractylodes macrocephala Koidz and others. Here, we report that CLT inhibited the ability of invasion and migration in metastatic breast cancer cells. Furthermore, CLT exhibited significant suppression on formation of lung metastatic foci of breast cancer in vivo. We next investigated the mechanism of CLT-induced metastasis inhibitory effects in breast cancer cells. A significant inhibition on activity and expression of MMP-9 and MMP-13 was observed. Moreover, data from western blotting, Runx 2 transcription factor assay and chromatin immunoprecipitation assay showed that binding ability of Runx 2 to sequences of the mmp-13 promoter was inhibited by CLT. Collectively, these findings suggested that the antimetastatic properties of CLT in breast cancer were due to the inhibition of MMPs, which might be associated with a downregulation of Runx 2 transcriptional activity.
\end{abstract}

Correspondence to: Dr Jianjiang Fu, Department of Pharmacology, School of Pharmacy, Jiangxi University of Traditional Chinese Medicine, Nanchang 330004, P.R. China

E-mail: jianjiang.fu@gmail.com

*Contributed equally

Key words: Codonolactone, invasion, migration, metastasis, Runx2, breast cancer

\section{Introduction}

Breast cancer is the most frequent malignant disease and the second cause of death from cancer in women in the United States (1). In most of these cases, relapse and consequent metastatic growth of cancer cells can occur at distant sites, including bone, lung, liver and brain (2-3). Metastasis to distant sites accounts for $>90 \%$ of breast cancer-related mortality (4). Despite its clinical importance, metastasis remains the most insidious aspect of breast cancer, and there are few successful treatments that directly target this stage. Therefore, better understanding of the complexities of the genetic and biochemical determinants of metastasis and developing effective therapies are urgently needed to reduce the morbidity and mortality from metastatic disease.

Natural products are still a major resource and have become increasingly important for new drug discoveries (5). Chloranthaceae, a basal angiosperm taxon, is composed of approximately 65-75 extant species (6) and over 10 medical herbs, most of which have antitumor associated usage (7). Sarcandra glabra Nakai, colloquially known as Caoshanhu, is a medical herb in Chloranthaceae that grows in the southern part of China (8). It is widely used as a kind of folk remedy for rheumatic arthralgia, bone fracture and other ailments (7). Tablets of $S$. glabra Nakai are clinically used as a well-known adjunctive therapy agent for leukemia, pancreatic cancer, and liver cancer, and can be found in the Pharmacopoeia of China (9). Unfortunately, the bioactive ingredients and their mechanisms of action are largely unknown. Interest has also been focused on the medical herb Chloranthus japonicas $(10,11)$, and the cytotoxicity of several sesquiterpenes isolated from the whole herb was evaluated. In addition, Kwon et al (12) reported that dimeric sesquiterpenoids isolated from this herb prevented monocyte adhesion to HUVECs via inhibiting the expression of cell adhesion molecules.

Led by these concepts, we hypothesized that antimetastatic activities may contribute to the antitumor related usage of plants in Chloranthaceae. Thus, we focused our interests 
on antimetastatic effects of four medical herbs in this genius, Sarcandra glabra Nakai, Chloranthus henryi Hemsl, Chloranthus fortune and Chloranthus multistachys Pei, which have close relationships and grow in Jiangxi Province, China (7). By invasion-inhibition-guide fractionation, we screened 263 constituents from these plants, including sesquiterpenes, diterpenes, chalcones, and sesquiterpene lactones (data not shown). Codonolactone (CLT), one of the sesquiterpene lactones isolated from C. henryi Hemsl, exhibited the strongest anti-invasive properties in vitro. In the present study, we report that CLT exhibited antimetastatic effects in metastatic breast cancer cells, and these effects may be mediated by inhibition of matrix metalloproteinases (MMPs) via downregulation of the transcriptional activity of Runx 2 .

\section{Materials and methods}

Materials. CLT was purchased from Shanghai PureOne Biotechnology (Shanghai, China; P0110) with a purity of $\geq 98.0 \%$, as determined by high-performance liquid chromatography. CLT was dissolved in $100 \%$ dimethyl sulfoxide (DMSO) as a stock solution and stored at $-20^{\circ} \mathrm{C}$. The final DMSO concentration did not exceed $0.1 \%$ throughout the study. The molecular structure of this sesquiterpene lactone is presented in Fig. 1. Batimastat (Bat), a broad spectrum MMP inhibitor, was purchased from Santa Cruz Biotechnology Inc. (SC-203833, Santa Cruz, CA, USA), and served as a positive control.

Cell culture. MDA-MB-231 human breast cancer cells, purchased from Cell Resource Center, Institute of Basic Medical Sciences, Chinese Academy of Medical Sciences, were maintained in Dulbecco's modified Eagle's medium (DMEM) supplemented with $10 \%$ fetal bovine serum (FBS; Gibco, Inc.), $100 \mathrm{IU} / \mathrm{ml}$ penicillin, and $100 \mu \mathrm{g} / \mathrm{ml}$ streptomycin (Invitrogen, Inc.) in a humidified incubator containing $5 \% \mathrm{CO}_{2}$ at $37^{\circ} \mathrm{C}$. Another breast cancer line, MDA-MB-157 was purchased from Sigma-Aldrich China (Beijing, China). MDA-MB-157 cells were maintained in L15 supplemented with $10 \%$ fetal bovine serum (FBS; Gibco, Inc.), 2 mM glutamine.

Animals and ethics statement. Five- to 6-week-old female NOD/SCID mice were purchased from Vital River Laboratories (Beijing, China) to establish an orthotopic xenograft tumor model and an experimental lung metastasis xenograft model. All the animal experiments were conducted in strict accordance with full ethical approval of the Animal Ethics Committees of Jiangxi University of Traditional Chinese Medicines. Surgery was performed under sodium pentobarbital anesthesia, and all efforts were made to minimize suffering.

In vitro invasive assay. In vitro invasive capacity of cancer cells was measured using the 48-well microchemotaxis system (AP 48, Neuro Probe, Gaithersburg, MD, USA). Briefly, a polycarbonate membrane with $8-\mu \mathrm{M}$ pore size was pre-coated with $5 \mu \mathrm{g}$ of fibronectin in a volume of $50 \mu \mathrm{l}$ on the rough (lower) surface. The Matrigel was diluted to $100 \mu \mathrm{g} / \mathrm{ml}$ with cold phosphate-buffered saline (PBS) and applied to the smooth (upper) surface of the filters ( $5 \mu \mathrm{g} /$ filter), and the filters were dried at room temperature. The lower compartments of the plates were filled with $30 \mu$ l DMEM containing $0.1 \%$ bovine serum albumin (BSA). Log-phase cells were harvested, washed three times with serum-free medium, and resuspended into a final concentration of $2 \times 10^{6} / \mathrm{ml}$ in DMEM with $0.1 \%$ BSA. Cell suspensions $(100 \mu \mathrm{l})$ containing $2 \times 10^{5}$ cells with or without CLT were added to the upper compartment and plates were incubated for $14 \mathrm{~h}$ in an incubator containing $5 \% \mathrm{CO}_{2}$ at $37^{\circ} \mathrm{C}$. The filters were fixed with methanol for $10 \mathrm{~min}$, stained with $0.5 \%$ crystal violet for $60 \mathrm{~min}$, and then washed with distilled water. The cells on the upper surface of the filters were removed by wiping with cotton swabs. The cells invading the lower surface of the filter through the Matrigel and filter were manually counted under light microscopy (Leica DM 4000), and images were captured by the Leica Application Suite (LAS V3.8.0).

In vitro migratory assay. In vitro migration of breast cancer cells was measured using Oris ${ }^{\mathrm{TM}}$ cell migration assay (Platypus Technologies, Madison, WI, USA) following the manufacturer's instructions. Log-phase cells were harvested, washed three times with serum-free medium, and resuspended into a final concentration of $4 \times 10^{6} / \mathrm{ml}$ in culture medium. Suspended cells $(100 \mu \mathrm{l})$ were pipetted into each test well through one of the side ports of the Oris ${ }^{\mathrm{TM}}$ Cell Seeding Stopper. The seeded plate containing the Oris Cell Seeding Stoppers in a humidified chamber $\left(37^{\circ} \mathrm{C}, 5 \% \mathrm{CO}_{2}\right)$ was incubated for $24 \mathrm{~h}$ to permit cell attachment. Using the Stopper Tool, all the stoppers were removed. Then, media was gently removed and the wells washed with PBS to remove the unattached cells. After that, $100 \mu \mathrm{l}$ of FBS-free media containing Calcein AM (final concentration $0.5 \mu \mathrm{g} / \mathrm{ml}$ ) was added, and the cells were incubated in a humidified chamber $\left(37^{\circ} \mathrm{C}, 0.5 \% \mathrm{CO}_{2}\right)$ for $40 \mathrm{~min}$. The images were captured under a fluorescence microscope (Leica DM 3000B), and these data served as the initial control. After fluorescence intensity examination, media were removed gently, and washed with PBS for 2 times. Then, $100 \mu \mathrm{l}$ of media containing vehicle, Bat or CLT was added, and the cells were incubated in a humidified chamber $\left(37^{\circ} \mathrm{C}, 0.5 \% \mathrm{CO}_{2}\right)$ for $24 \mathrm{~h}$. At the end of treatment, data were obtained with the methods mentioned above.

Cell proliferation assay. Cell proliferation was assayed by MTT assay. Briefly, log phase cells were seeded in 96-well plates $24 \mathrm{~h}$ before initiation of treatment with CLT. The vehicletreated cells served as controls. Three duplicate wells were set up in each sample. After incubation with CLT, Bat or vehicle for $48 \mathrm{~h}$, cells were incubated with 3-(4, 5-dimethylthiazol2-yl)-2,5-diphenyltetrazolium bromide (MTT, Sigma-Aldrich) (final concentration $0.5 \mathrm{mg} / \mathrm{ml}$ ) for $4 \mathrm{~h}$ at $37^{\circ} \mathrm{C}$. The media was carefully removed from each well and $200 \mu \mathrm{l}$ of DMSO was added. The plates were gently agitated and optical absorbance at OD570 $\mathrm{nm}$ and OD450 $\mathrm{nm}$ was determined using an ELx800 microplate reader (BioTek, Winooski, VT, USA). Vehicle only-treated cells served as the indicator of $100 \%$ cell viability. Each experiment was repeated three times.

In vivo growth assay. For the growth assay, MDA-MB-231 cell xenografts were established by injection of $1 \times 10^{7}$ cells to the mammary fat pad in NOD/SCID mice. After 3 weeks of growth, the tumors were removed and chopped into $1 \times 1 \times 1-\mathrm{mm}$ 
pieces, and the pieces were then implanted at the mammary fat pad of mice. The mice bearing tumor chunks were randomly divided into three groups: control, CLT (25 mg/kg per day) treatment group, and CLT ( $75 \mathrm{mg} / \mathrm{kg}$ per day) treatment group. Forty-eight hours later, CLT was administered by gavage on a regimen of 6-day dosing per week for 5 weeks. Tumor growth was assessed by measuring the length and width of tumors with electronic calipers every 3-4 days continuously. Volumes were calculated using the formula: (length) $\mathrm{x}$ (width) ${ }^{2} / 2$.

In vivo experimental metastasis assay and survival assay. To establish experimental lung metastasis xenograft model, $2 \times 10^{6}$ MDA-MB-231 cells in $200 \mu \mathrm{l}$ normal saline were injected through tail vein into NOD/SCID mice. The mice were also divided into control, CLT ( $25 \mathrm{mg} / \mathrm{kg}$ per day) treatment group, and CLT $(75 \mathrm{mg} / \mathrm{kg}$ per day) treatment group. CLT treatment began $24 \mathrm{~h}$ after tumor injection. After 8-week treatment, the formation of metastatic foci in lung tissues was measured by nest reverse-transcription polymerase chain reaction (RT-PCR). The mice were euthanized and dissected, and lungs were snap-frozen in liquid nitrogen. Total RNA was isolated from each lung using the QIAshredder and RNeasy Protect Mini kit (Qiagen) for detection of human cytokeratin 19 (ck19) by nested PCR.

For the survival assay, the experimental lung metastasis xenograft model was established using the same method as in vivo experimental metastasis assay, but the observation period was 120 days.

RNA isolation and nested RT-PCR. Nested RT-PCR was used to detect expression of the human ck19 gene in lung tissues of tumor-bearing mice. Total RNA was isolated from each lung using the QIAshredder and RNeasy Protect Mini kit (Qiagen). Ck19 was reverse transcribed in the presence of $2 \mu \mathrm{l}$ enzyme mix, $10 \mu \mathrm{l}$ RNA and outer primer for $30 \mathrm{~min}$ at $50^{\circ} \mathrm{C}$, according to the manufacturer's instructions (Qiagen OneStep RT-PCR kit, Qiagen). After Taq polymerase activation for 15 min at $95^{\circ} \mathrm{C}$, samples were amplified for 37 cycles at $94^{\circ} \mathrm{C}$ for $45 \mathrm{sec}, 58^{\circ} \mathrm{C}$ for $45 \mathrm{sec}$ and $72^{\circ} \mathrm{C}$ for $90 \mathrm{sec}$. Target primers for amplifying ck19 (outer primer) were designed using Primer Designer (Scientific \& Educational Software Version 2.0). The forward primer for ck19 (GenBank no. BC010409) was 5'-cca cgt cgt cet tcg gag gcc-3' (64-84 bp) and reverse primer was 5'-gttc cgt ctc aaa ctt ggt tc-3' (529-549 bp). After a final extension for $10 \mathrm{~min}$ at $72^{\circ} \mathrm{C}$, the RT-PCR product was further subjected to nested PCR using the Qiagen Multiplex PCR kit (Qiagen). The inner primers were forward 5'-tac agc cac tac tac acg acc atc c-3' (432-456 bp) and reverse 5'-gga caa tcc tgg agt tct caa tg-3' (488-510 bp). The nested PCR profile was as follows: $3 \mathrm{~min}$ at $94^{\circ} \mathrm{C}$, followed by 35 three-step cycles of $45 \mathrm{sec}$ at $94^{\circ} \mathrm{C}, 45 \mathrm{sec}$ at $60^{\circ} \mathrm{C}$ and $1 \mathrm{~min}$ at $72^{\circ} \mathrm{C}$. PCR reactions were subjected to final extension at $72^{\circ} \mathrm{C}$ for 10 min. Nested RT-PCR analysis was performed using the Mastercycler gradient (Eppendorf). The $\beta$-actin gene was used as an internal control for standardization, and the primers, Tm, and cycles are the same as reported (13). The nested PCR product was separated by $4 \%$ agarose (UltraPure ${ }^{\mathrm{TM}}$ Agarose, Invitrogen) gel electrophoresis, and the gels were viewed by UV transillumination and photographed by the UVP EC3 gel imaging system.
FRET-based MMP activity assay. Activity of MMPs was also measured by the SensoLyte ${ }^{\circledR} 570$ Generic MMP assay kit (AnaSpec, Fremont, CA, USA). This kit provides a FRETbased method to detect the activities of a variety of MMPs including MMP-1, 2, 3, 7, 8, 9, 10, 11, 12, 13 and 14. It uses 5-FAM (fluorophore) and QXL520 ${ }^{\mathrm{TM}}$ (quencher) labeled FRET peptide substrates for continuous measurement of MMP activity. In an intact FRET peptide, the fluorescence of 5-FAM is quenched by SensoLyte. Upon the cleavage of FRET peptide by MMPs, the fluorescence of 5-FAM is recovered. Analyses were performed according to the manufacturer's instructions. Briefly, supernatants of breast cancer cells were collected after incubation with or without CLT for $12 \mathrm{~h}$. The MMPs in supernatants were activated by incubation with 4-aminophenylmercuric acetate for $1 \mathrm{~h}$ at $37^{\circ} \mathrm{C}$. MMPs containing $50 \mu \mathrm{l}$ of samples and $50 \mu \mathrm{l}$ MMP substrate solution were added into a 96-well plate. The reagents were mixed by shaking the plate gently for $30 \mathrm{sec}$. After a $50-\mathrm{min}$ incubation period at $37^{\circ} \mathrm{C}$, the reaction was stopped by adding stop solution, and fluorescence intensity was measured by a multilabel counter $\left(\right.$ Victor $^{3 \mathrm{TM}}$, Perkin-Elmer, Waltham, MA, USA) at Ex/Em=540/575 nm.

Western blot analysis. Cells were rinsed twice with PBS, and total proteins were extracted in $500 \mu \mathrm{l}$ lysis buffer. Aliquots of whole cell lysates were added to $10 \%$ SDS-PAGE and then transferred to Hybond nitro-blotting membranes. The membranes were blocked with $3 \%$ BSA in Tris-buffered saline containing $0.5 \mathrm{ml} / 1$ Tween-20 and then incubated with primary antibodies against MMP-9 (Santa Cruz, SC-6840), MMP-13 (Santa Cruz, SC-30073), Runx2 (Santa Cruz, SC-10758) and phospho-Runx2 (Abgent, AP3559a), followed by incubation with horseradish peroxidase (HRP)conjugated secondary antibodies. Immunoreactive proteins were detected using an enhanced chemiluminescence kit (Millipore). $\beta$-actin (Santa Cruz, SC-130301) served as an internal control.

Immunohistochemistry. MMP-13 in tumor tissues was determined by immunostaining. Formalin-fixed, paraffin-embedded MDA-MB-231 tumor tissues were cut into 3- $\mu$ m thick sections and mounted on poly-lysine coated slides. After dewaxing and hydration, antigen retrieval was performed by incubation with Proteinase $\mathrm{K}$ at $37^{\circ} \mathrm{C}$ for $20 \mathrm{~min}$. After three 5-min rinses in PBS $(0.01 \mathrm{~mol} / \mathrm{l})$, the sections were treated with $3 \% \mathrm{H}_{2} \mathrm{O}_{2}$ in methanol for 10 min to block endogenous peroxidase activity per the manufacturer's instructions (Zhongshan Golden-bridge Biotech., Beijing, China). Sections were then blocked with $10 \%$ normal goat serum (Nichirei, Tokyo, Japan) for $30 \mathrm{~min}$ at room temperature. The tissues were incubated with primary antibodies against MMP-13 (Santa Cruz, SC-30073; 1:50) or PBS at $4^{\circ} \mathrm{C}$ overnight. After three rinses in PBS, the slides were incubated with HRP-labeled IgG (SC-2004, Santa Cruz) for $60 \mathrm{~min}$, and then peroxidase activity was detected by the AEC Substrate System (ab64252, Abcam). All the slides were examined by light microscopy (Leica DM 4000), and images were captured using the Leica Application Suite (LAS V3.8.0).

Runx2 transcription factor assay. Runx 2 activity of nuclear extracts was detected using the TransAM ${ }^{\mathrm{TM}}$ AML-3/Runx2 kit (Active Motif North America, Carlsbad, CA, USA) following 


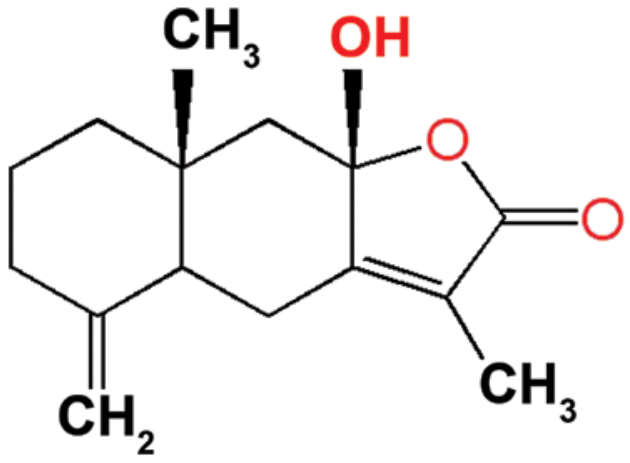

Codonolactone

Figure 1. Molecular structure of Codonolactone. the manufacturer's instructions. Cell extracts were prepared using the Nuclear Extract kit (Active Motif) with breast cancer cells that were treated with either CLT or DMSO for $12 \mathrm{~h}$. Then, $20 \mu \mathrm{l}$ of extracts diluted in complete lysis buffer and containing $15 \mu \mathrm{g}$ nuclear extract were added into a 96-well plate. This plate immobilizes oligonucleotides containing Runx 2 consensus binding sites. Saos- 2 nuclear extract served as a positive control for Runx 2 activation, and $20 \mu \mathrm{l}$ complete lysis buffer served as the blank. The wild-type consensus oligonucleotide was provided as a competitor for Runx2 binding to monitor the specificity of the assay. After 1-h incubation at room temperature, the plate was washed three times with washing buffer. Diluted primary antibody $(100 \mu \mathrm{l})$ was added into wells and incubated for $1 \mathrm{~h}$ at room temperature
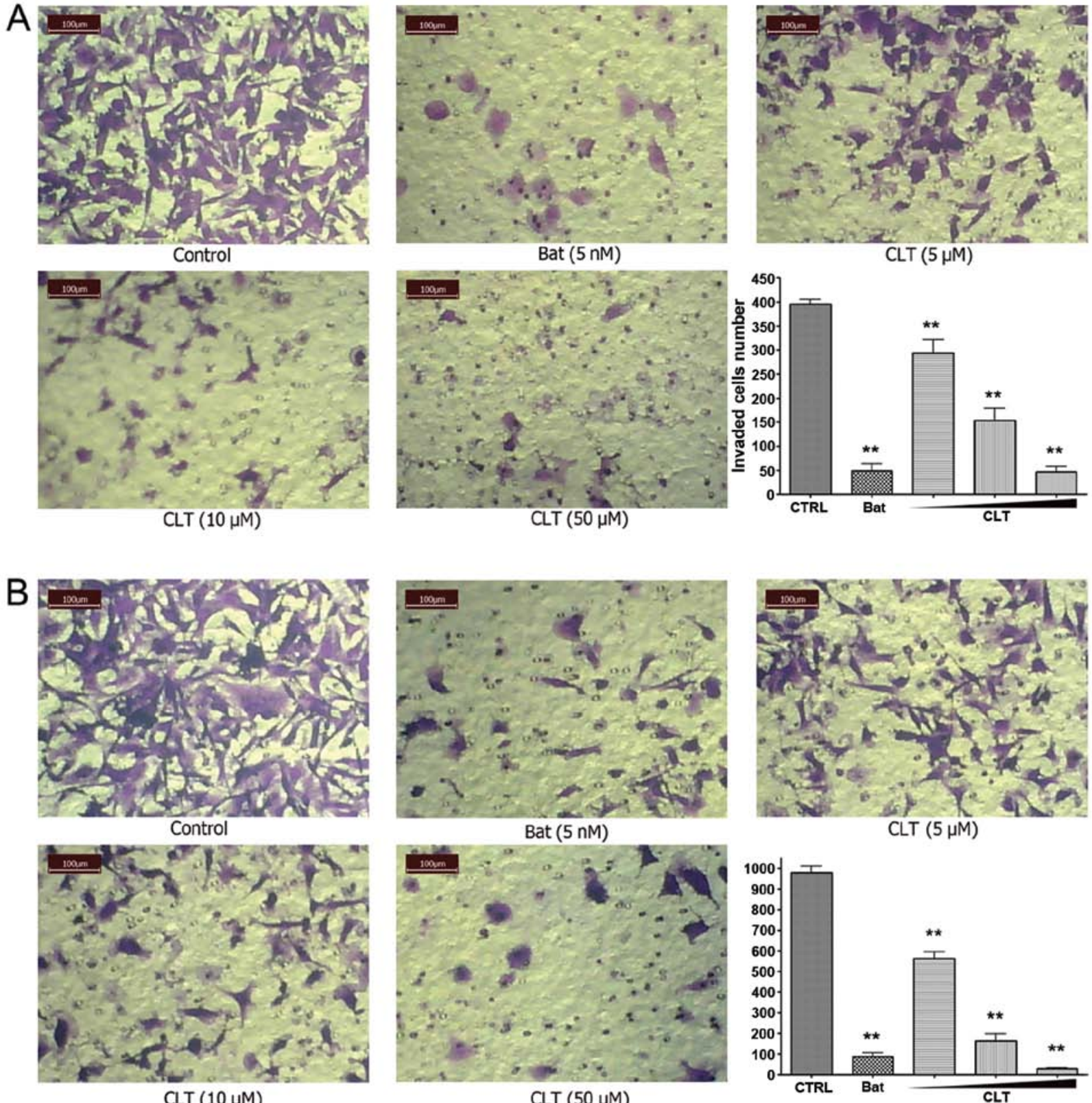

Figure 2. In vitro invasion of breast cancer cells is significantly affected by CLT in a dose-dependent manner. (A) CLT inhibited the invasion of MDA-MB-231 cells in the AP48 chamber. Five fields were counted for each chamber, and each concentration was repeated in three chambers. (B) Effects of CLT on invasion of MDA-MB-157 cells. In vitro invasion assay was carried out as described in Materials and methods. ${ }^{* *} \mathrm{p}<0.01$ compared with CTRL. Vehicle (DMSO) was used as a CTRL, and Bat served as the positive CTRL. Each assay was performed in triplicate. CLT, Codonolactone; Bat, Batimastat; CTRL, control. 
without agitation. After three washes, HRP-labeled secondary antibody was added and incubated for $1 \mathrm{~h}$ at room temperature. Then, $100 \mu \mathrm{l}$ developing solution was added to initiate the color reaction. After $100 \mu \mathrm{l}$ stop solution was added, the absorbance was measured within $5 \mathrm{~min}$ at $450 \mathrm{~nm}$ with a reference wavelength of $655 \mathrm{~nm}$ using an ELx800 microplate reader (BioTek).

Chromatin immunoprecipitation assay. Chromatin immunoprecipitation assay (ChIP) was performed using the Agarose ChIP kit as described in the manufacturer's instructions (Pierce $^{\mathrm{TM}}$ Agarose ChIP kit, Thermo Scientific). Briefly, crosslinking was performed for $10 \mathrm{~min}$ using formaldehyde (final concentration 1\%). Cross-linked cells then were lysed by lysis buffer containing protease inhibitors. To obtain DNA fragments with an average size of $0.3 \mathrm{~kb}$, micrococcal nuclease was added and incubated for $5 \mathrm{~min}$. Protein-DNA complexes were immunoprecipitated using anti-Runx2 antibody (Santa Cruz, SC-10758). Normal rabbit IgG served as a negative control and anti-RNA polymerase II antibody served as positive control. After DNA recovery, purified DNA was subjected to PCR amplification on the Mastercycler gradient (Eppendorf) using Phusion ${ }^{\circledR}$ High-Fidelity PCR kit. The primers designed to amplify one of the Runx 2 binding regions (-1531 bp: acaccaa) in human mmp-13 promoter (GeneBank no. NM_002427) were forward, 5'-aac ttg gta gct ttt atg gtg g-3' and reverse, 5'-gcc tct tca tca gat aat aag gg-3'. The PCR profile was as follows: $15 \mathrm{~min}$ at $95^{\circ} \mathrm{C}$, followed by 30 three-step cycles of $15 \mathrm{sec}$ at $95^{\circ} \mathrm{C}, 30 \mathrm{sec}$ at $57^{\circ} \mathrm{C}$ and $30 \mathrm{sec}$ at $72^{\circ} \mathrm{C}$. PCR reactions were subjected to final extension at $72^{\circ} \mathrm{C}$ for $10 \mathrm{~min}$. PCR analysis was performed using the Mastercycler gradient (Eppendorf). Aliquots of total DNA before immunoprecipitation were saved as input, and these input lysates were also processed as above. All ChIP assays were performed at least three times, and the most representative results are illustrated in the figures.

Statistical analysis. The data are presented as mean \pm SD and were analyzed with SPSS for Windows (13.0) software program (Chicago, IL, USA). Comparison among different groups was carried out by one-way analysis of variance (the one-way ANOVA). Analysis of number of mice bearing metastatic foci in lung was performed by $\chi^{2}$ test. The difference between the means was considered statistically significant at $\mathrm{p}<0.05$.

\section{Results}

CLT inhibits invasion and migration of breast cancer cells in vitro. First, we evaluated the effects of CLT on invasion using a reconstituted basement membrane system. We used fibronectin as a chemo-attractant on the lower surface of a polycarbonate membrane. Matrigel was plated on the upper surface of the polycarbonate membrane to mimic the basement membrane. After 14-h incubation, CLT significantly blocked the trans-membrane invasion of MDA-MB-231 cells, with an $88.37 \%$ inhibition rate at $50 \mu \mathrm{M}$ (Fig. 2A). Invasion of MDA-MB-157 cells was also significantly blocked by CLT (Fig. 2B), with inhibitions of $42.58,83.32$ and $97.15 \%$ at concentrations of 5,10 and $50 \mu \mathrm{M}$, respectively. Next, the effect of CLT on cell migration in vitro was tested using Oris cell migration system. As shown in Fig. 3, after 24-h incubation, CLT inhibited the migration of breast cancer cells in a dose-dependent manner. These results indicate that CLT could inhibit the invasion, migration of MDA-MB-231 and MDA-MB-157 cells concentration-dependently.

CLT slightly suppresses tumor growth in vitro and in vivo, but induces a significant increase of overall survival and a considerable inhibitory effect on formation of lung metastatic foci in NOD/SCID mice. To assess the effect of CLT on the proliferation of MDA-MB-231 and MDA-MB-157 cells, we first observed the growth status of these cells treated by CLT or vehicle using the MTT assay. The growth curve is shown in Fig. 4A. The proliferation of MDA-MB-231 and MDA-MB-157 cells was inhibited slightly after CLT exposure for $48 \mathrm{~h}$. The inhibitory rate on MDA-MB-231 cell growth was $22.19 \%$ when the cells were treated with $100 \mu \mathrm{M} \mathrm{CLT}$ for $48 \mathrm{~h}$, and the inhibition rate was $20.60 \%$ on MDA-MB-157 cells. Next, we examined the inhibitory effects of CLT in vivo. An orthotopic xenograft model was established by injection of MDA-MB-231 cells into mammary fat pads of NOD/SCID mice. The tumor volume curve during this study is shown in Fig. 4B. Compared with vehicle-treated animals, CLT administered at $75 \mathrm{mg} / \mathrm{kg} /$ day caused very slight inhibition on primary tumor growth.

Next, the antimetastatic effects of CLT were evaluated using experimental metastasis model. MDA-MB-231 cells spreading to the lung of cancer cell injected NOD/SCID mice were assessed by detecting the expression of human ck19 using nested PCR. The most typical bands and the statistical results of relative densitometric units of lung tissues in each group are illustrated in Fig. 4C. CLT treatment showed overt inhibition of human ck-19 expression in lung tissues of mice in a dose-dependent manner. Compared to control group, the average inhibition rate of CLT treatment group (25 and $75 \mathrm{mg} / \mathrm{kg} /$ day) was 17.67 and $65.56 \%$, respectively (Fig. 4C). These results suggest that the primary effects of CLT were not in the growth inhibition of tumor cells, but in metastatic suppression.

To better understand the overall benefits of CLT, we evaluated the effect of CLT on the overall survival of tumor cell-injected mice after treatment for 120 days. As shown in Fig. 4D, both CLT-treated groups showed a prolonged survival and an increasing survival time dose-dependently. Animals in the control group died 63 days and onwards after tumor inoculation, and the median survival period is 78 days. There is only 1 of the total 20 surviving mice at the endpoint under this condition. However, for groups treated with CLT 25 and $75 \mathrm{mg} / \mathrm{kg} / \mathrm{day}$, the median survival days ranged from 94 to 100 days, and there were still two and four survivals at the endpoint, respectively. These data indicated that tumor bearing mice may benefit from CLT treatment, which may be associated with its antimetastatic effects.

CLT inhibits MMP activity and expression in MDA-MB-231 and $M D A-M B-157$ cells. Extensive work on the mechanisms of tumor invasion and metastasis has identified MMPs as key players in these events (14). Therefore, we next examined the effects of CLT on MMPs in breast cancer cells. Fig. 5 shows the effects of CLT on MMPs in the metastatic breast cancer cells. Using a FRET-based analysis, we found that CLT 

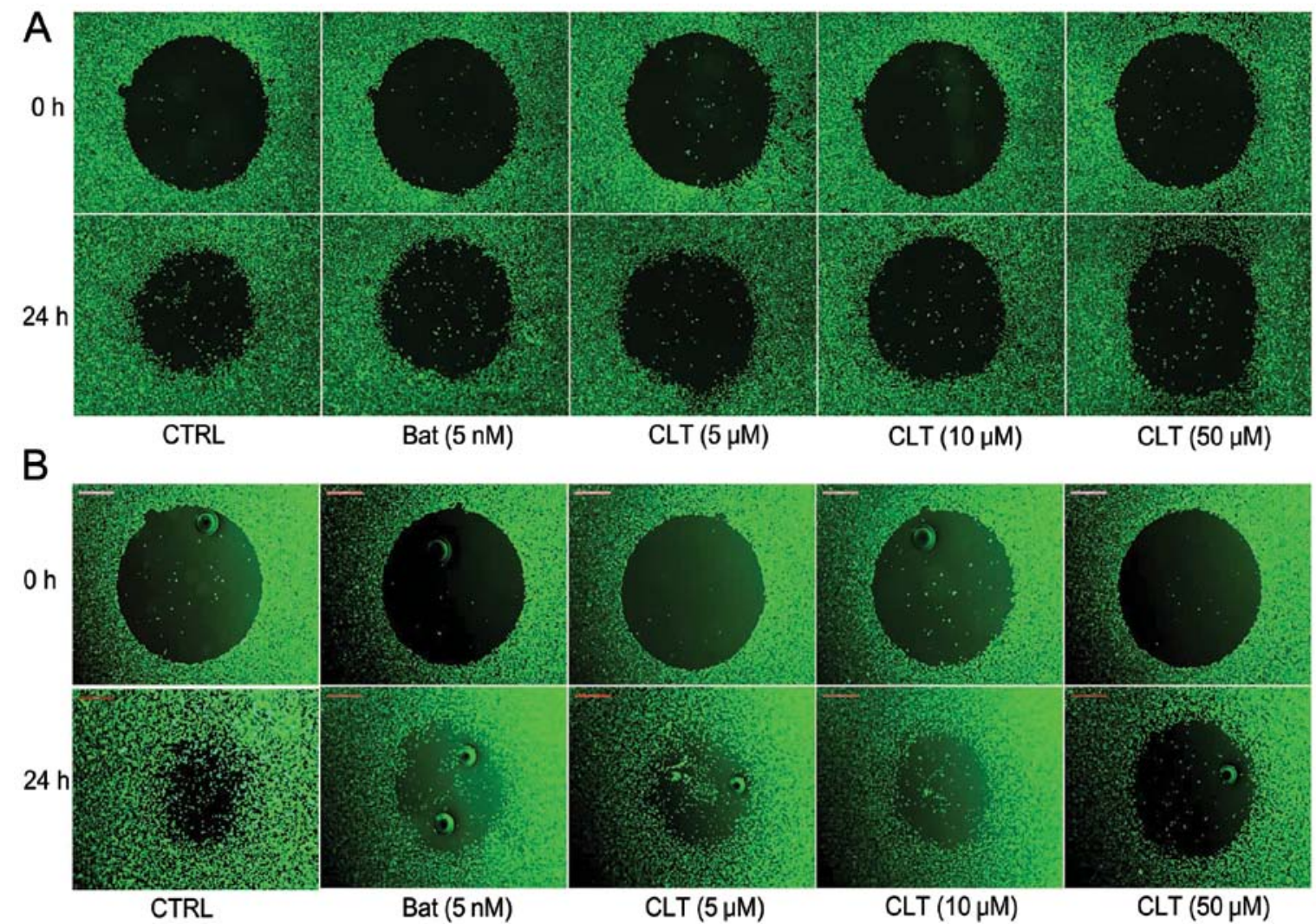

Figure 3. Effects of CLT on migration of breast cancer cells. (A) CLT inhibited the migration of MDA-MB-231 cells in Oris cell migration system. (B) Effects of CLT on in vitro migration of MDA-MB-157 cells. In vitro migration assay was carried out as described in Materials and methods. Vehicle (DMSO) was used as a CTRL and Bat served as the positive CTRL. Each assay was performed in triplicate. CLT, Codonolactone; Bat, Batimastat; CTRL, control.

A
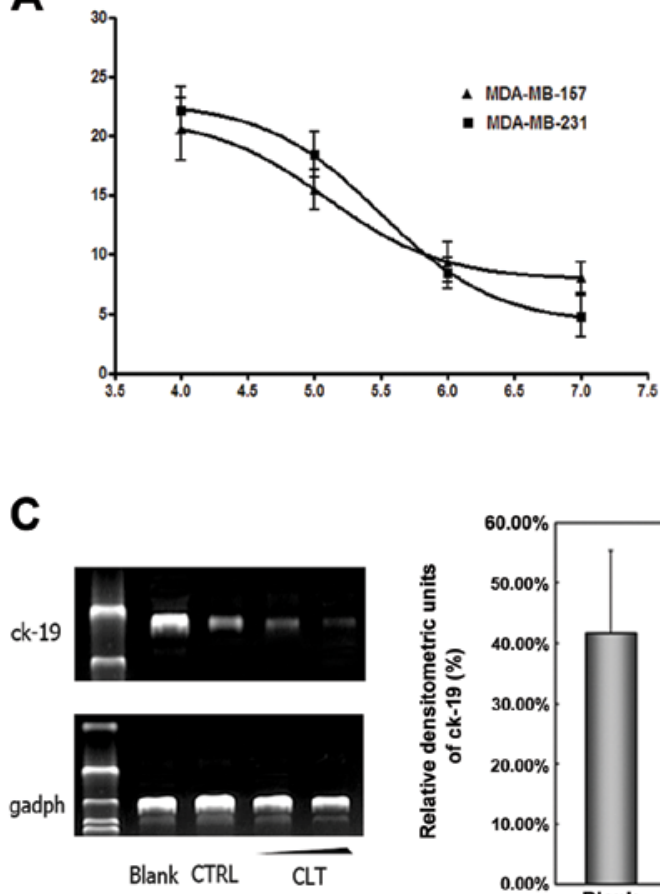

B

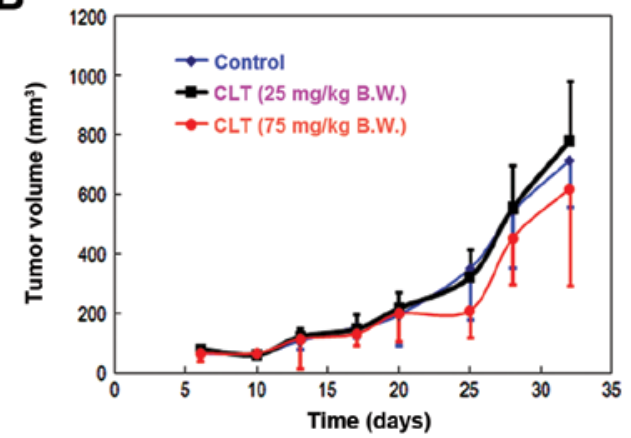

D

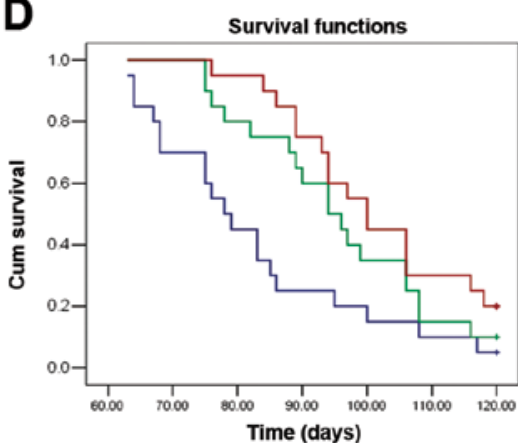

Figure 4. CLT slightly suppresses tumor growth in vitro and in vivo, but significantly increases overall survival and inhibits formation of lung metastatic foci in NOD/SCID mice. CLT showed a slight suppression on cell growth in vitro (A) and in vivo (B). However, there was a significant inhibitory effect on the formation of lung metastatic foci (C) and a considerable increase of overall survival (D) in NOD/SCID mice. Orthotopic xenograft models and in vivo metastatic models were established according to Materials and methods. Lung metastatic foci were determined by nested RT-PCR. Total RNA was extracted from each lung tissue. RNA isolated from cultured MDA-MB-231 cells served as a blank and RNA extracted from the CTRL group served as CTRL. ${ }^{*} \mathrm{p}<0.05 ;{ }^{* * *} \mathrm{p}<0.01$ compared with CTRL. CLT, Codonolactone; CTRL, control. 
A

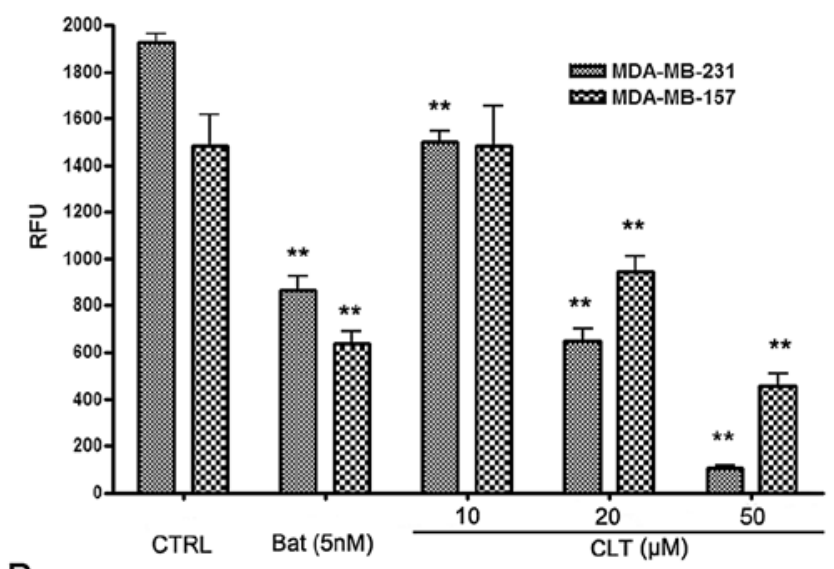

B

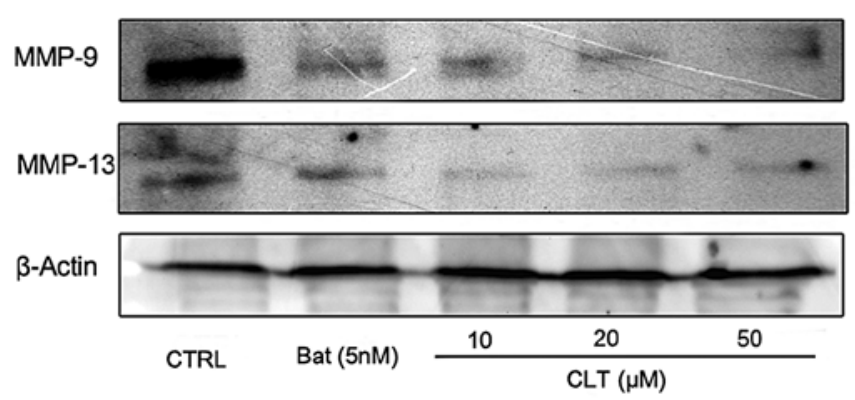

C

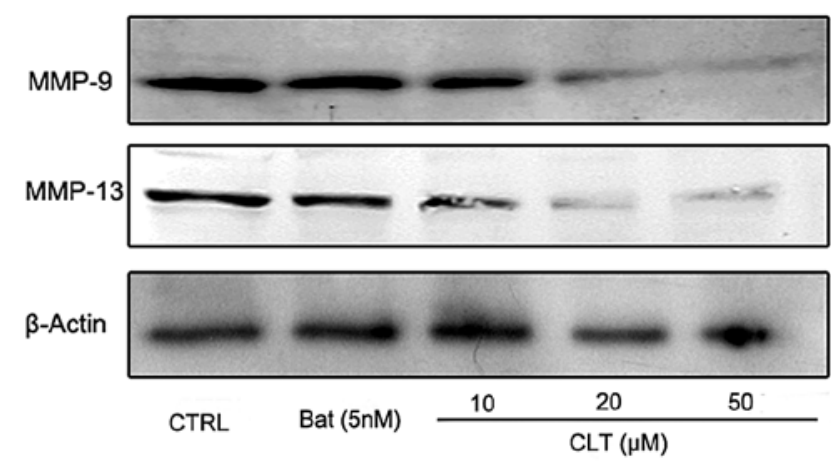

Figure 5. Effects of CLT on activity of matrix metalloproteinases in metastatic breast cancer cells. (A) MMP protein activity was detected by the FRET-based MMP activity assay kit. (B) CLT inhibited MMP-9 and MMP-13 expression in MDA-MB-231 cells as detected by western blotting. (C) CLT also inhibited MMP-9 and MMP-13 expression in MDA-MB-157 cells. Vehicle (DMSO) was used as CTRL, and batimastat (Bat) served as a positive control. ${ }^{* * *} \mathrm{p}<0.01$ compared with CTRL. Each assay was performed in triplicate at minimum. CLT, Codonolactone; Bat, Batimastat; CTRL, control.

exhibited significant suppression of FRET substrate cleavage of MMPs in a dose-dependent manner (Fig. 5A). To further identify whether CLT inhibited the functional activity or expression of MMPs in the breast cancer cells, we analyzed the expression of MMP-9 and MMP-13 in both culture cells and tumor tissues treated with CLT. As shown in Fig. 4B and $\mathrm{C}$, CLT significantly decreased MMP-13 expression in MDA-MB-231 and MDA-MB-157 breast cancer cells in a dose-dependent manner. Then, IHC assay was involved in present study to confirm the effects of CLT on MMP-13 expression. It was also observed that CLT inhibited MMP-13 expression in tumor tissues (Fig. 6 and Table I). These data
Table I. Semiquantitative analysis of MMP-13 in MDA-MB-231 tumor tissues.

\begin{tabular}{lccc}
\hline Group & $\begin{array}{c}\text { Dose } \\
(\mathrm{mg} / \mathrm{kg})\end{array}$ & $\begin{array}{c}\text { Relative area of MMP-13 } \\
\text { immunostaining }(\%)^{\mathrm{a}}\end{array}$ & $\begin{array}{c}\text { Inhibition } \\
\text { rate }(\%)\end{array}$ \\
\hline Blank & - & $1.62 \pm 0.69$ & - \\
CTRL & - & $34.89 \pm 10.40$ & - \\
CLT & 25 & $25.61 \pm 5.72$ & 26.60 \\
& 75 & $8.66 \pm 2.12^{\mathrm{b}}$ & 75.18
\end{tabular}

Orthotopic xenograft model was established as described in Materials and methods. Expression of MMP-13 in tumor tissues was detected by immunohistochemistry as described in Materials and methods.

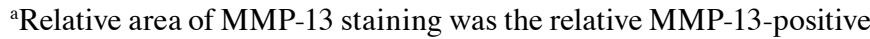
area of the total area. Data are expressed as mean $\pm \mathrm{SD}(\mathrm{n}=3) .{ }^{\mathrm{b}} \mathrm{p}<0.01$ compared with control. CLT, codonolactone; CTRL, control.

suggested that the antimetastatic properties of CLT may be due to downregulation of expression of MMP-9 and MMP-13 in breast cancer.

CLT suppresses activation of Runx2 and impedes its binding to sequences in the mmp-13 promoter in MDA-MB-231 cells. From current concepts, we understood that Runx2 is a 'master' transcriptional factor of metastatic growth of breast cancer cells. Several genes required for the formation of metastatic foci, including MMP-9, MMP-13, BSP, OPN, VEGF, are targets of this transcriptional factor (15). So we examined the levels of total Runx2 and phospho-Runx2 in MDA-MB-231 cells. As shown in Fig. 7A, CLT had no effect on total Runx2 expression, but caused a significant decrease of phosphoRunx2 expression. These results indicate that CLT-induced downregulation of MMP-13 may be associated with the suppression of Runx2 activation.

To confirm the western blot results, Runx 2 transcription factor assay and ChIP assay were designed to evaluate the effects of CLT on Runx2 activities in MDA-MB-231 cells. An enzyme-linked immunoabsorbent assay (ELISA)-based kit for the Runx2 transcription factor was used to analyze the effects of CLT on Runx2. MDA-MB-231 nuclear extracts incubated with CLT or vehicle were prepared, and binding activity between Runx 2 with its target sequence was determined. The results showed that CLT downregulated the binding activity of Runx 2 in a dose-dependent manner (Fig. 7B). Using the ChIP assay, we found that binding of Runx 2 to one of the Runx2 binding domains $(-1,531 \mathrm{bp}$ : aca cca a) in the mmp-13 promoter region was inhibited by CLT (Fig. 7C). These findings suggested that the inhibitory effect of CLT on MMP-13 was caused by CLT downregulation of Runx 2 binding to the mmp-13 promoter, which may be associated with suppression of Runx 2 phosphorylation.

\section{Discussion}

Breast cancer is the leading cause of death in women with cancer, and most of the deaths are due to the distant spread of cancer cells (1), and growth in distant organs of cancer cells 


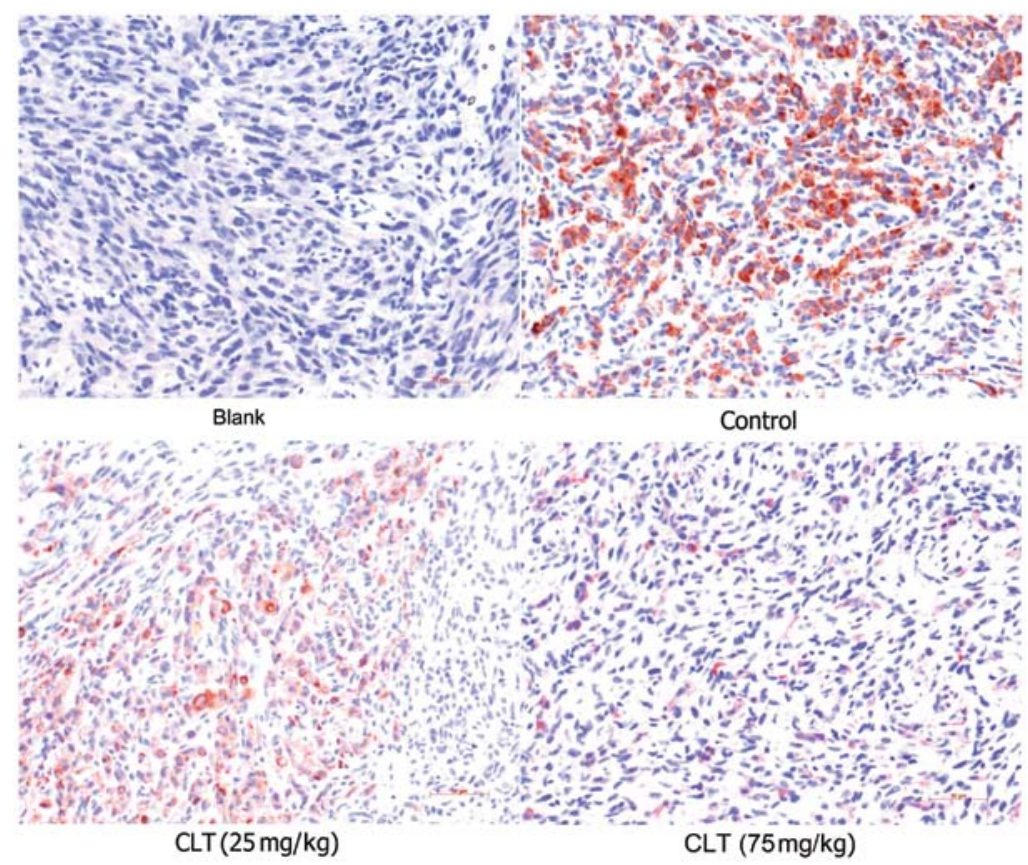

Figure 6. CLT attenuated expression of MMP-13 in tumor tissues. Expression of MMP-13 in tumor tissues was detected by immunohistochemistry as described in Materials and methods. The most representative results are illustrated in the figures. Blank, no primary antibody incubated.

A

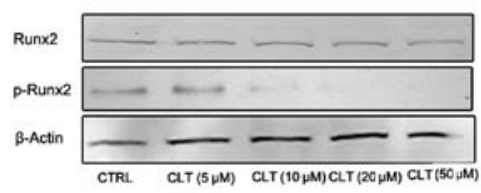

B

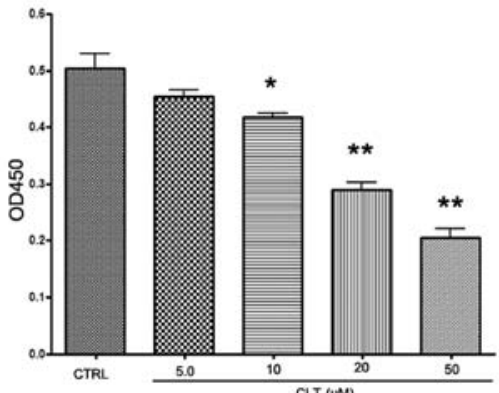

C

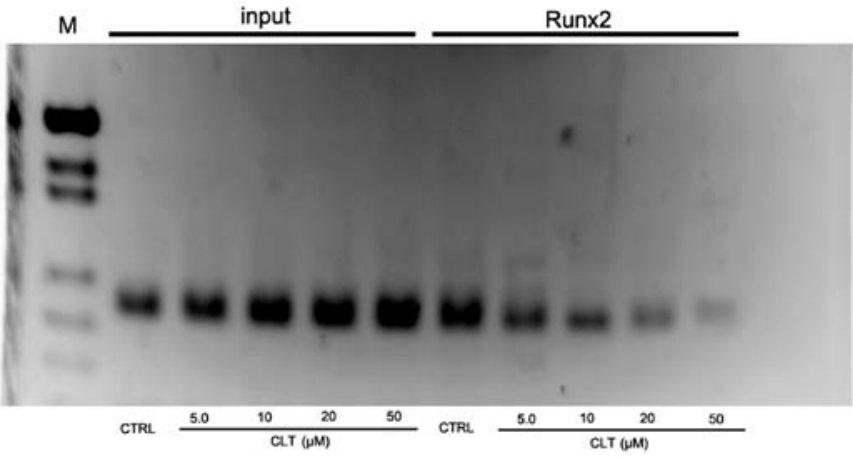

Figure 7. CLT inhibits MMP-13 protein activity and expression through downregulating activation of Runx2 transcription factor in MDA-MB-231 cells. CLT suppressed phosphorylation and transcription factor activity of Runx2 as detected by western blotting (A). A Runx2 transcription factor assay kit (B) and chromatin immunoprecipitation assay (C) was used to confirm the effects of CLT on Runx2. Lane M, DNA marker. Vehicle (DMSO) was used as a control. ${ }^{*} \mathrm{p}<0.05 ;{ }^{* *} \mathrm{p}<0.01$ compared with CTRL. CLT, Codonolactone; CTRL, control. (metastasis), is the most insidious aspect of this malignancy. Better understanding of metastasis and developing effective therapies are urgently required. On the other hand, natural products are still the main resource of new drug development (5), and therefore our interests have been focused on identifying new potential therapeutic agents from Chinese herbal medicine. Here we report that CLT, a sesquiterpene lactone which can be found in several Chinese herbal medicines, suppressed invasion, migration of MDA-MB-231 and MDA-MB-157 cells. Furthermore, these in vitro effects were confirmed by in vivo data. CLT exhibited significant suppression of formation of lung metastatic foci and improvement on total survival of tumor-bearing mice.

MMPs are a family of structurally and functionally related zinc-dependent endopeptidases. To date, 23 human MMPs, including 17 soluble, secreted enzymes and six membraneassociated enzymes have been identified (14). These enzymes are involved in a wide range of physiological and pathological processes, such as embryonic development, wound healing, tumor growth, invasion and metastasis (16). During the past 30 years, the role of MMPs in human cancer has been widely investigated. These enzymes participate in the proteolysis of the extracellular matrix, modulation of cell adhesion, migration (17), and epithelial to mesenchymal transition (EMT) (18), processing of growth factors, and tumor-induced angiogenesis. In breast cancer, intensive data suggested critical roles for MMPs in both breast cancer initiation and progression (17). Based on this evidence, we examined the effects of CLT on MMP-9 and MMP-13. Our results showed that CLT induced a significant suppression of the activity and expression of MMP-9 and MMP-13. These data suggested that CLT attenuated the metastatic properties of breast cancer cells by MMPs inhibition.

Runx2, also named PEBP2 $\alpha \mathrm{A} / \mathrm{AML} 3 / \mathrm{Cbfa} 1$, is a critical transcription factor for osteoblastic differentiation 
and skeletal morphogenesis (19-21). This protein belongs to the Runx family encoding proteins homologous to Drosophila Runt and has a conserved Runt DNA-binding domain. Originally, Runx 2 was found to act as a master regulatory factor in skeletal development (22). This transcriptional factor acts as a master regulatory factor involved in skeletal gene expression by binding DNA as a monomer or, with more affinity, as a subunit of a heterodimeric complex (19-22). To date, extensive evidence shows a close association between Runx 2 and breast cancer metastasis, and this transcriptional factor is becoming a potential target of novel antimetastatic agents and diagnostic approaches to breast cancer control (15,23-25). Runx2 is highly expressed in both breast cancer cell lines which have the tendency to form metastatic foci and breast primary tumors. In breast cancer cells, several genes that correlated with the occurrence of skeletal metastasis have been shown to be regulated by Runx2, such as bsp and opn $(26,27)$. However, the functions of Runx 2 on tumor metastasis are far beyond colonizing bone. Recently, it was reported that Runx 2 is not only a novel transcriptional regulator of tumorinduced angiogenesis (28) but also plays a critical role in cancer cell induced EMT (29).

Runx2 is also involved in the regulation of MMP in metastatic breast cancer cells. Jimenez et al first found that MMP-13, also named Collagenase-3, was highly expressed in MDA-MB-231 cells, and that it was one of target genes of Runx 2 (30). These observations were demonstrated by Selvamurugan and colleagues $(31,32)$. Studies showed that the runt domain (RD) binding site and Runx2 were required for maximal constitutive and basal expression of MMP-13 in MDA-MB-231 cells. Furthermore, the ChIP assay confirmed two Runx 2 binding sites in the mmp-13 promoter, and these sites are occupied by Runx2. Pratap et al investigated the role of Runx 2 in the regulation of the promoter of MMP-9, in MDA-MB-231 and MCF-7 cells. MMP-9 was found to be another direct target of Runx2 in metastatic breast cancer cells, and the modulation of Runx 2 activity by either forced expression or RNA interference directly affected MMP-9 expression and the invasive properties of metastatic cancer cells (33). Thus, Runx 2 acts a master transcription factor of MMPs expression. Therefore, we wondered whether the inhibitory effects of CLT on MMP-9 and MMP-13 were associated with the downregulation of Runx 2 activities. Our findings from western blotting demonstrated that CLT significantly inhibited the expression of p-Runx2, indicating that the transcriptional ability of Runx 2 is impaired by CLT. To confirm these findings, we used an ELISAbased Runx 2 transcription factor assay in this study, and the results revealed that the interaction between Runx 2 and its target sequences was significantly inhibited by CLT. On the other hand, the interaction of Runx 2 and mmp-13 promoter was evaluated by ChIP. It was found that binding between Runx 2 and mmp-13 promoter was impaired significantly by CLT.

In conclusion, here we report that CLT, a sesquiterpene lactone, impairs the metastatic potential of breast cancer, and the inhibitory effects are due to its ability to reduce the expression of MMP-9 and MMP-13, targets of the Runx2 transcription factor. These effects may be associated with inhibition of transcriptional activity of Runx 2 .

\section{Acknowledgements}

This study was supported by Grants from the National Natural Science Foundation of China (grant nos. 30860375, 81160530 and 81260656), Key Research Project from the Ministry of Education of China (Grant Number: 211091), and the Natural Science Foundation of Jiangxi Province (grant no. 2010GQY0147).

\section{References}

1. Siegel R, Naishadham D and Jemal A: Cancer Statistics, 2013. CA Cancer J Clin 63: 11-30, 2013.

2. Leong SP, Cady B, Jablons DM, et al: Clinical patterns of metastasis. Cancer Metastasis Rev 25: 221-232, 2006.

3. Stevanovic A, Lee P and Wilcken N: Metastatic breast cancer. Aust Fam Physician 35: 309-312, 2006.

4. Liu S, Goldstein RH, Scepansky EM, et al: Inhibition of rhoassociated kinase signaling prevents breast cancer metastasis to human bone. Cancer Res 69: 8742-8751, 2009.

5. Newman DJ: Natural products as leads to potential drugs: an old process or the new hope for drug discovery? J Med Chem 51: 2589-2599, 2008.

6. Hristova K, Lam M, Feild T, et al: Transmitting tissue ECM distribution and composition, and pollen germinability in Sarcandra glabra and Chloranthus japonicus (Chloranthaceae). Ann Bot 96: 779-791, 2005.

7. Editorial Committee of the Administration Bureau of Traditional Chinese Medicine: genus Chloranthaceae. In: Chinese Materia Medica (zhonghua Bencao). Shanghai Science \& Technology Press, Shanghai, pp2051-2061, 1998.

8. Pan C, Xu H, Peng H, et al: Resource investigation and exploitable foreground of Sarcandra glabra. Zhong Yao Cai 27: 556-557, 2004.

9. National Committee of Pharmacopoeia: Pharmacopoeia of People's Republic of China. 2010 ed. China Medical Science Press, Beijing, pp842, 2010.

10. Wang QH, Kuang HX, Yang BY, et al: Sesquiterpenes from Chloranthus japonicus. J Nat Prod 74: 16-20, 2011.

11. Zhang M, Wang JS, Wang PR, et al: Sesquiterpenes from the aerial part of Chloranthus japonicus and their cytotoxicities. Fitoterapia 83: 1604-1609, 2012.

12. Kwon OE, Lee HS, Lee SW, et al: Dimeric sesquiterpenoids isolated from Chloranthus japonicus inhibited the expression of cell adhesion molecules. J Ethnoparmacol 104: 270-277, 2006.

13. Fu J, Ding Y, Huang D, et al: The retinoid X receptor-selective ligand, LGD1069, inhibits tumor-induced angiogenesis via suppression of VEGF in human non-small cell lung cancer. Cancer Lett 248: 153-163, 2007.

14. Chambers AF and Matrisian LM: Changing views of the role of matrix metalloproteinases in metastasis. J Natl Cancer Inst 89: 1260-1270, 1997

15. Inman CK and Shore P: The osteoblast transcription factor Runx 2 is expressed in mammary epithelial cells and mediates osteopontin expression. J Biol Chem 278: 48684-48689, 2003.

16. Roy R, Yang J and Moses MA: Matrix metalloproteinases as novel biomarkers and potential therapeutic targets in human cancer. J Clin Oncol 27: 5287-5297, 2009.

17. Duffy MJ, Maguire TM, Hill A, et al: Metalloproteinases: role in breast carcinogenesis, invasion and metastasis. Breast Cancer Res 2: 252-257, 2000.

18. Radisky ES and Radisky DC: Matrix metalloproteinase-induced epithelial-mesenchymal transition in breast cancer. J Mammary Gland Biol Neoplasia 15: 201-212, 2010.

19. Lian JB, Stein JL, Stein GS, et al: Runx2/Cbfa1 functions: diverse regulation of gene transcription by chromatin remodeling and co-regulatory protein interactions. Connect Tissue Res 44: $141-148,2003$.

20. Karsenty G: Role of Cbfa1 in osteoblast differentiation and function. Semin Cell Dev Biol 11: 343-346, 2000.

21. Komori T: Runx2, a multifunctional transcription factor in skeletal development. J Cell Biochem 87: 1-8, 2002.

22. Stein GS, Lian JB, A. van Wijnen J, et al: Runx 2 control of organization, assembly and activity of the regulatory machinery for skeletal gene expression. Oncogene 23: 4315-4329, 2004. 
23. Inman CK, Li N and Shore P: Oct-1 counteracts autoinhibition of Runx2 DNA binding to form a novel Runx $2 /$ Oct- 1 complex on the promoter of the mammary gland-specific gene beta-casein. Mol Cell Biol 25: 3182-3193, 2005.

24. Barnes GL, Javed A, Waller SM, et al: Osteoblast-related transcription factors Runx2 (Cbfa1/AML3) and MSX2 mediate the expression of bone sialoprotein in human metastatic breast cancer cells. Cancer Res 63: 2631-2637, 2003.

25. Shore P: A role for Runx 2 in normal mammary gland and breast cancer bone metastasis. J Cell Biochem 96: 484-489, 2005.

26. Ganss B, Kim RH and Sodek J: Bone sialoprotein. Crit Rev Oral Biol Med 10: 79-98, 1999.

27. Bellahcène $\mathrm{A}$ and Castronovo V: Expression of bone matrix proteins in human breast cancer: potential roles in microcalcification formation and in the genesis of bone metastases. Bull Cancer 84: 17-24, 1997.

28. Sun L, Vitolo M and Passaniti A: Runt-related gene 2 in endothelial cells: inducible expression and specific regulation of cell migration and invasion. Cancer Res 61: 4994-5001, 2001.
29. Chimge NO, Baniwal SK, Little GH, et al: Regulation of breast cancer metastasis by Runx 2 and estrogen signaling: the role of SNAI2. Breast Cancer Res 13: R127, 2011.

30. Jiménez MJ, Balbín M, López JM, et al: Collagenase 3 is a target of Cbfa1, a transcription factor of the runt gene family involved in bone formation. Mol Cell Biol 19: 4431-4442, 1999.

31. Selvamurugan N and Partridge NC: Constitutive expression and regulation of collagenase-3 in human breast cancer cells. Mol Cell Biol Res Commun 3: 218-223, 2000.

32. Selvamurugan N, Kwok S and Partridge NC: $\operatorname{Smad} 3$ interacts with JunB and Cbfa1/Runx2 for transforming growth factor-beta1stimulated collagenase-3 expression in human breast cancer cells. J Biol Chem 279: 27764-27773, 2004.

33. Pratap J, Javed A, Languino LR, et al: The Runx2 osteogenic transcription factor regulates matrix metalloproteinase 9 in bone metastatic cancer cells and controls cell invasion. Mol Cell Biol 25: 8581-8591, 2005. 